\title{
BMJ Open Factors associated with the uptake of seasonal influenza vaccination in older and younger adults: a large, population- based survey in Beijing, China
}

Shuangsheng Wu, ${ }^{1,2}$ Jianting Su, ${ }^{1,2}$ Peng Yang, ${ }^{1,2,3}$ Haiyan Zhang, ${ }^{4}$ Hongjun Li, ${ }^{5}$ Yanhui Chu, ${ }^{6}$ Weiyu Hua, ${ }^{7}$ Chao Li, ${ }^{7}$ Yaqing Tang, ${ }^{8}$ Quanyi Wang ${ }^{1,2}$

To cite: Wu S, Su J, Yang P, et al. Factors associated with the uptake of seasonal influenza vaccination in older and younger adults: a large, population-based survey in Beijing, China. BMJ Open 2017;7:e017459. doi:10.1136/ bmjopen-2017-017459

- Prepublication history for this paper is available online. To view these files please visit the journal online (http://dx.doi org/10.1136/bmjopen-2017017459).

SW and JS contributed equally.

Received 24 April 2017 Revised 11 August 2017 Accepted 21 August 2017

CrossMark

For numbered affiliations see end of article.

\section{Correspondence to}

Peng Yang;

yangpengcdc@163.com and Dr

Quanyi Wang;

bjcdcxm@126.com

\section{ABSTRACT}

Objectives The present study aimed to estimate the influenza vaccination coverage rate in Beijing, China, and identify its determinants in older and younger adults. Methods A survey was conducted among Chinese adults using a self-administered, anonymous questionnaire in May-June 2015. The main outcome was seasonal influenza vaccination uptake. Multivariate logistic regression models were performed to identify factors associated with uptake.

Results A total of 7106 participants completed the questionnaire. The overall coverage rate was $20.6 \%$ (95\% Cl $19.7 \%$ to $21.5 \%$ ) in the $2014 / 2015$ influenza season. Lower education (older adults: OR 1.6; $95 \% \mathrm{Cl}$ 1.2 to 2.1 ; younger adults: $\mathrm{OR} 1.9 ; 95 \% \mathrm{Cl} 1.4$ to 2.6 ), having a chronic illness (older adults: OR $1.9 ; 95 \% \mathrm{Cl}$ 1.5 to 2.4; younger adults: $\mathrm{OR} 1.4 ; 95 \% \mathrm{Cl} 1.2$ to 1.7 ) and recommendations from healthcare workers (older adults: OR 5.4; $95 \% \mathrm{Cl} 3.9$ to 7.4 ; younger adults: OR 4.5; $95 \% \mathrm{Cl} 3.7$ to 5.4 ) were positively associated with uptake; perceived side effects of vaccination had a negative impact (older adults: OR 0.6; $95 \% \mathrm{Cl} 0.4$ to 0.7 ; younger adults: OR $0.8 ; 95 \% \mathrm{Cl} 0.7$ to 1.0$)$. Perceived susceptibility to influenza (OR $1.5 ; 95 \% \mathrm{Cl} 1.2$ to 2.0 ) and awareness of the free influenza vaccine policy (OR 1.9; $95 \% \mathrm{Cl} 1.2$ to 2.9) were only associated with vaccine uptake in older adults, while perceived effectiveness of vaccination (OR 2.2; 95\% $\mathrm{Cl} 1.7$ to 2.8 ) was only a predictor for younger adults. Older adults were more likely to receive recommendations from healthcare professionals and perceive the severity of seasonal influenza, and less likely to worry about side effects of vaccination.

Conclusion The influenza vaccination coverage rate was relatively low in Beijing. Apart from free vaccinations for older adults, age disparity in the rate between older and younger adults (48.7\% vs $16.0 \%)$ may be explained by differing professional recommendations and public perceptions. Vaccination campaigns targeting increasing professional recommendations and public perceptions should be implemented in the coming years.

\section{INTRODUCTION}

Seasonal influenza is a weighty public health problem that causes substantial mortality and morbidity, especially among older people and

\section{Strengths and limitations of this study}

- Strengths of this study were the large sample size of 7106 participants and high response rate of $98.7 \%$, indicating high representation and reliability in the findings.

- Self-reported influenza vaccination uptake may result in recall bias.

- Causal relationships could not be established because of the cross-sectional design.

others with high-risk conditions. ${ }^{1}$ Worldwide, annual epidemics are estimated to result in about 3-5 million cases of severe illness and 250000-500000 deaths. ${ }^{2}$ Vaccination is an effective way to minimise influenza-related mortality and morbidity ${ }^{3-5}$; many public health organisations, including the China Centers for Disease Prevention and Control, have recommended all people $>6$ months of age, particularly those at high risk, receive the influenza vaccine annually. ${ }^{6}$ Although seasonal vaccination has been recommended for many years, a global study of seasonal influenza vaccine dose distribution found that coverage remains low in many countries, especially low-and middle-income countries. ${ }^{7}$ People in most regions of China bear the full cost of the vaccine; this led to the very low $1.5 \%-2.2 \%$ coverage rate between 2004 and $2014 .^{8}$

The Chinese capital of Beijing has a population of about 20 million. Abundant and convenient transportation and high population density make the city an easily affected host for both seasonal and pandemic influenza. ${ }^{9}$ Since 2007, the Beijing government, ahead of governments in most cities in China, has provided free influenza vaccines to people aged $\geq 60$ years, and for students in primary or middle schools, between September and November of each year. Available data show 
the policy greatly increased the vaccine uptake rate in the qualifying population. ${ }^{10}$ Therefore, Beijing's experience can serve to inform future government-funded reimbursement policies for seasonal influenza vaccination in other regions of China, as well as in other developing countries.

Our previous study in Beijing estimated the influenza vaccination coverage rate of the general population at $16.7 \%$ in $2008 / 2009,16.9 \%$ in $2010 / 2011$ and $21.8 \%$ during the 2009 pandemic. The rates were much higher than in other regions of China, yet lower than those of Western countries. ${ }^{11}$ Coverage was much higher among older adults than younger adults in Beijing in 2010, but lower than that among older adults in other countries with similar policies. ${ }^{12}$ The influencing factors associated with uptake of the influenza vaccine need to be determined in order to improve coverage. Meanwhile, previous studies in Beijing only determined demographics factors for uptake. ${ }^{11}$ Apart from a free vaccination policy and demographics, many other factors, including perceptions of personal risk, disease severity, effectiveness and side effects of vaccination, and the related impact of healthcare workers may also affect uptake. $^{1314}$

In the present study, we performed a population-based survey for ongoing assessment of influenza vaccination uptake in Beijing's general population. The study aimed to (1) estimate coverage rates among older adults aged $\geq 60$ years and younger adults aged $<60$ years in the 2014/2015 influenza season; (2) identify the factors-including demographics factors, public perceptions and impact of healthcare workers-associated with uptake; and (3) find the reasons for age-related differences in the coverage rate between younger and older adults.

\section{METHODS}

\section{Study participants}

This study employed a population-based survey in the Beijing metropolitan area. The target population was Chinese adults living in Beijing for longer than half a year. The function $\mathrm{n}=\mu_{\alpha}{ }^{2} \times \bar{\Phi} \times(1-\bar{\varpi}) / \delta^{2} \times$ deff was used to calculate the sample size of each subgroup. We estimated a sample size of 576 participants per subgroup, based on $\mu_{\alpha}=1.96$, influenza vaccination rate $(\varpi)=50 \%$, maximum permissible error $(\delta)=0.1 \Phi$ and design effect of complex sampling (deff $)=1.5$. Considering area of residence (urban or suburban), and different age groups (18-29, $30-39,40-49,50-59$ and $\geq 60$ years) and a no-response rate of $25 \%$, the optimal sample size for the present study was 7200 (576 participants per subgroup $\times 10$ subgroups $\times 1.25$ ). Participants were randomly selected from 150 survey locations that were the same as in the previous study. ${ }^{11}$ All adults in each survey location were numbered, and then 48 were randomly selected to meet the sample size requirement.

\section{Data collection}

The survey was conducted using a self-administered, anonymous questionnaire in May-June 2015. The questionnaire was in Chinese and consisted of five sections: receiving seasonal influenza vaccine in the 2014/2015 influenza season, having a fever within the past year, recommendations from healthcare workers, public perceptions towards influenza and its vaccine, and demographics. (1) History of receiving the vaccine was assessed using the yes/no question: 'Did you receive the seasonal influenza vaccine during the previous season (2014/2015)?' (2) Recommendations from healthcare workers were assessed using: 'Did healthcare workers recommend the seasonal influenza vaccine to you during the previous season (2014/2015)?' (3) History of having a fever within the past year was assessed using: 'Have you had a fever within the past year?' (4) To assess public perceptions towards influenza and its vaccine, the following yes/no questions were asked: 'Do you think influenza is a serious disease?' 'Are you afraid of catching influenza during an epidemic?' 'Do you think the vaccine can prevent influenza infection?' 'Are you scared of the vaccine's side effects?' 'Do you know about the free influenza vaccine policy in Beijing?' (5) Regarding demographics information, the participants were asked to report their sex (female or male), age (continuous), highest educational attainment (primary school or none, junior high school, senior high school, 3-year college graduate or above), residence (urban or suburban), monthly income per capita $(0-2000$ or $>2000$ yuan; US\$100 is equivalent to approximately 680 yuan), family population which refers to the number of people living in the home (continuous), number of children in the family (continuous) and history of chronic illness (yes or no). The average monthly income of Beijing residents was 3659 and 1685 yuan in urban and suburban areas, respectively. All questions were developed based on measures adapted from our previous study and from the existing literature..$^{11} 1314$

Local healthcare workers performed all interviews. Before each questionnaire was distributed, all investigators were required to explain to respondents the purpose, procedures and confidentiality agreement for the study, and written informed consent was accordingly obtained. In most cases, the participants completed the questionnaires independently. The investigators read and explained the questionnaires to candidates who were unable to sufficiently understand them.

\section{Statistical analysis}

The main outcome was seasonal influenza vaccination uptake in the 2014/2015 influenza season. Descriptive analysis was performed to generate frequency distributions of the survey variables, and differences between the subgroups were tested using Pearson's $\chi^{2}$ test. The tables list the numbers of participants for whom missing data were reported. Weighted analysis was conducted to calculate weighted coverage rates, accounting for age, sex and residence of those in the Beijing population, as 
reported in the 2010 Census of Beijing. ${ }^{15}$ Multivariate logistic regression models were performed to examine the factors associated with uptake of the vaccine, and the OR and $95 \%$ CIs were used as measures of association. All statistical tests were two-sided, with $\mathrm{p}<0.05$ considered statistically significant. Data entry was performed using Epidata software V.3.1 (The EpiData Association, Odense, Denmark), while data analyses were performed using SPSS V.20.0 (IBM Corporation, New York, USA).

\section{RESULTS}

\section{Demographics of study participants}

Of the 7200 people recruited for the study, 7106 completed the survey. The characteristics of the participants are presented in table 1 . Of these, $50.9 \%$ were female $(\mathrm{n}=3614)$ and $48.8 \%$ lived in urban areas $(\mathrm{n}=3468)$. The distribution of age was as follows: 18-29: $20.5 \%(\mathrm{n}=1450)$; 30-39: 20.1\% ( $\mathrm{n}=1424)$; 40-49: 20.6\% ( $\mathrm{n}=1461)$; 50-59: $19.6 \%(\mathrm{n}=1391)$; and $\geq 60$ years: $19.2 \%(\mathrm{n}=1362)$.

\section{Weighted coverage rates of seasonal influenza vaccine}

The weighted coverage rate of the seasonal influenza vaccine was $20.6 \%$ (95\% CI $19.7 \%$ to $21.5 \%$ ) among adults in Beijing during the 2014/2015 influenza season. Regarding the difference between age groups, the coverage rates were $48.7 \%$ (95\% CI $46.0 \%$ to $51.4 \%$ ) and $16.0 \%$ (95\% CI $14.1 \%$ to $17.9 \%$ ) in older adults aged $\geq 60$ years and younger adults aged $<60$ years, respectively.

\section{Univariate analysis of variables affecting seasonal influenza vaccination uptake}

Overall, 1610 (22.7\%) participants reported having received seasonal influenza vaccine during the previous season $(2014 / 2015)$. The rates did not differ by sex $(p=0.541)$ or residence $(p=0.275)$. The rate among older adults aged $\geq 60$ years was significantly higher $(\mathrm{p}<0.001)$. Rates decreased with increasing education levels, from $43.8 \%$ of participants with no or primary school education to $18.7 \%$ of those who were 3-year college graduates or higher $(\mathrm{p}<0.001)$. The significantly different rates were observed between the two income categories $(27.0 \%$ vs $21.0 \%, \mathrm{p}<0.001)$. Rates decreased with family population, from $28.7 \%$ of participants whose family population was one or two people to $21.5 \%$ of those whose family population were six or more people $(\mathrm{p}<0.001)$. The rates were significantly higher among people with a chronic illness $(35.5 \%$ vs $17.1 \%, \mathrm{p}<0.001)$ or who had a fever in the past year $(27.5 \%$ vs $22 \%, \mathrm{p}<0.001)$. Participants who received recommendations from healthcare workers were more likely to be vaccinated $(33.0 \%$ vs $8.0 \%, \mathrm{p}<0.001)$. Those with awareness of the severity of the disease, susceptibility to the disease, effectiveness of the vaccination and the free influenza vaccine policy in Beijing were more likely to be vaccinated $(\mathrm{p}<0.001)$, while those concerned about side effects of vaccination were less likely $(\mathrm{p}=0.006)$ (table 1$)$.
Multiple logistic regression analysis for the factors associated with seasonal influenza vaccination uptake

As shown in table 2, the results of multiple logistic regression analysis indicated that lower education (older adults: OR $1.6 ; 95 \%$ CI 1.2 to 2.1 ; younger adults: OR $1.9 ; 95 \%$ CI 1.4 to 2.6), having a chronic illness (older adults: OR 1.9; $95 \% \mathrm{CI} 1.5$ to 2.4; younger adults: OR $1.4 ; 95 \% \mathrm{CI} 1.2$ to 1.7) and recommendations from healthcare workers (older adults: OR 5.4; $95 \%$ CI 3.9 to 7.4; younger adults: OR $4.5 ; 95 \%$ CI 3.7 to 5.4 ) were positively associated with influenza vaccination uptake; perceived side effects of vaccination had a negative impact (older adults: OR 0.6; $95 \%$ CI 0.4 to 0.7 ; younger adults: OR $0.8 ; 95 \%$ CI 0.7 to 1.0). Regarding age-related differences, perceived susceptibility to influenza (OR 1.5; 95\% CI 1.2 to 2.0 ) and awareness of the free influenza vaccine policy (OR 1.9; $95 \%$ CI 1.2 to 2.9 ) were only associated with vaccine uptake in older adults, while perceived effectiveness of vaccination (OR 2.2; 95\% CI 1.7 to 2.8) was only a predictor for younger adults.

Comparison between age groups regarding disease history, recommendations from healthcare workers and public perceptions

Pearson's $\chi^{2}$ tests indicated that older adults aged $\geq 60$ years were more likely to receive recommendations from healthcare workers $(73.1 \%$ vs $55.2 \%, \mathrm{p}<0.001)$, have a chronic illness $(66.2 \%$ vs $22.1 \%, \mathrm{p}<0.001)$, perceive severity of the disease $(55.9 \%$ vs $51.2 \%, \mathrm{p}=0.002)$ and be aware of the free influenza vaccine policy in Beijing $(87.1 \%$ vs $83.0 \%, \mathrm{p}<0.001)$. They were less likely to be concerned about side effects of the vaccination $(54.0 \%$ vs $61.6 \%, \mathrm{p}<0.001)$. For both older and younger adults, compared with more highly educated participants, lower-educated participants with primary school education or below were less likely to report fear of side effects (older adults: $46.2 \%$ vs $57.6 \%$, p $<0.001$; younger adults: $54.4 \%$ vs $62.0 \%, \mathrm{p}<0.001)$ and that the influenza vaccine could prevent infection (older adults: $78.9 \%$ vs $84.1 \%$, $\mathrm{p}=0.021$; younger adults: $75.6 \%$ vs $81.7 \%, \mathrm{p}=0.007$ ), whereas they were more likely to report influenza was a serious disease (older adults: $73.2 \%$ vs $65.9 \%, \mathrm{p}=0.007$; younger adults: $73.6 \%$ vs $58.4 \%, \mathrm{p}<0.001)$. Moreover, lower-educated older adults reported receiving recommendations from healthcare workers more frequently than more highly educated participants $(77.5 \%$ vs $70.9 \%$, $\mathrm{p}=0.012$ ). For younger adults, lower-educated participants were more likely to have a chronic illness $(39.4 \%$ vs $20.7 \%, \mathrm{p}<0.001)$.

\section{DISCUSSION}

In this study, we performed a population-based survey for ongoing assessment of influenza vaccination uptake in the general population of Beijing. The survey showed vaccination coverage rates in the general population of Beijing were 20.6\% during the 2014/2015 influenza season, which was nearly the same as in certain European 
Open Access

Table 1 Univariate analysis of variables affecting seasonal influenza vaccination uptake in Beijing during the 2014/2015 influenza season

\begin{tabular}{|c|c|c|c|c|c|c|}
\hline \multirow[b]{2}{*}{ Variables } & \multirow{2}{*}{$\begin{array}{l}\begin{array}{l}\text { Total } \\
\text { participants }\end{array} \\
\text { N }\end{array}$} & \multicolumn{2}{|c|}{ Vaccinated participants } & \multicolumn{3}{|c|}{ Unvaccinated participants } \\
\hline & & $\mathbf{n}$ & $\%^{*}$ & n & $\% *$ & p Value \\
\hline \multicolumn{7}{|l|}{ Sex } \\
\hline Male & 3484 & 778 & 22.3 & 2706 & 77.7 & 0.541 \\
\hline Female & 3614 & 829 & 22.9 & 2785 & 77.1 & \\
\hline Missing & 8 & 3 & & 5 & & \\
\hline \multicolumn{7}{|l|}{ Age (years) } \\
\hline $18-29$ & 1450 & 216 & 14.9 & 1234 & 85.1 & $<0.001$ \\
\hline $30-39$ & 1424 & 212 & 14.9 & 1212 & 85.1 & \\
\hline $40-49$ & 1461 & 203 & 13.9 & 1258 & 86.1 & \\
\hline $50-59$ & 1391 & 302 & 21.7 & 1089 & 78.3 & \\
\hline$\geq 60$ & 1362 & 672 & 49.3 & 690 & 50.7 & \\
\hline Missing & 18 & 5 & & 13 & & \\
\hline \multicolumn{7}{|l|}{ Highest education } \\
\hline Primary school or none & 730 & 320 & 43.8 & 410 & 56.2 & $<0.001$ \\
\hline Junior high school & 1850 & 418 & 22.6 & 1432 & 77.4 & \\
\hline Senior high school & 2167 & 422 & 19.5 & 1745 & 80.5 & \\
\hline 3-year college graduate or above & 2334 & 437 & 18.7 & 1897 & 81.3 & \\
\hline Missing & 25 & 13 & & 12 & & \\
\hline \multicolumn{7}{|l|}{ Residence } \\
\hline Suburban & 3638 & 805 & 22.1 & 2833 & 77.9 & 0.275 \\
\hline Urban & 3468 & 805 & 23.2 & 2663 & 76.8 & \\
\hline Missing & 0 & 0 & & 0 & & \\
\hline \multicolumn{7}{|l|}{ Monthly income per capita (yuan)‡ } \\
\hline $0-2000$ & 1887 & 509 & 27.0 & 1378 & 73.0 & $<0.001$ \\
\hline$>2000$ & 5023 & 1056 & 21.0 & 3967 & 79.0 & \\
\hline Missing & 196 & 45 & & 151 & & \\
\hline \multicolumn{7}{|l|}{ Family population } \\
\hline $1-2$ & 547 & 157 & 28.7 & 390 & 71.3 & $<0.001$ \\
\hline 3 & 1831 & 487 & 26.6 & 1344 & 73.4 & \\
\hline 4 & 2415 & 458 & 19.0 & 1957 & 81.0 & \\
\hline 5 & 1179 & 264 & 22.4 & 915 & 77.6 & \\
\hline$\geq 6$ & 1130 & 243 & 21.5 & 887 & 78.5 & \\
\hline Missing & 4 & 1 & & 3 & & \\
\hline \multicolumn{7}{|l|}{ Number of children in the family } \\
\hline 0 & 3915 & 959 & 24.5 & 2956 & 75.5 & $<0.001$ \\
\hline 1 & 2795 & 548 & 19.6 & 2247 & 80.4 & \\
\hline$\geq 2$ & 300 & 65 & 21.7 & 235 & 78.3 & \\
\hline Missing & 96 & 38 & & 58 & & \\
\hline \multicolumn{7}{|l|}{ History of chronic illness } \\
\hline Yes & 2149 & 763 & 35.5 & 1386 & 64.5 & $<0.001$ \\
\hline No & 4957 & 847 & 17.1 & 4110 & 82.9 & \\
\hline Missing & 0 & 0 & & 0 & & \\
\hline
\end{tabular}

Continued 
Table 1 Continued

\begin{tabular}{|c|c|c|c|c|c|c|}
\hline \multirow[b]{2}{*}{ Variables } & \multirow{2}{*}{$\begin{array}{l}\begin{array}{l}\text { Total } \\
\text { participants }\end{array} \\
\mathbf{N} \\
\end{array}$} & \multicolumn{2}{|c|}{ Vaccinated participants } & \multicolumn{2}{|c|}{ Unvaccinated participants } & \multirow[b]{2}{*}{ p Value } \\
\hline & & $\mathbf{n}$ & $\%^{*}$ & $\mathbf{n}$ & $\%^{*}$ & \\
\hline Yes & 894 & 246 & 27.5 & 648 & 72.5 & $<0.001$ \\
\hline No & 6194 & 1363 & 22.0 & 4831 & 78.0 & \\
\hline Missing & 18 & 1 & & 17 & & \\
\hline \multicolumn{7}{|c|}{ Recommendations from healthcare workers } \\
\hline Yes & 4168 & 1376 & 33.0 & 2792 & 67.0 & $<0.001$ \\
\hline No & 2938 & 234 & 8.0 & 2704 & 92.0 & \\
\hline Missing & 0 & 0 & & 0 & & \\
\hline \multicolumn{7}{|c|}{ Perceived severity of the disease (influenza is a serious disease) } \\
\hline Yes & 4328 & 1044 & 24.1 & 3284 & 75.9 & $<0.001$ \\
\hline No & 2778 & 566 & 20.4 & 2212 & 79.6 & \\
\hline Missing & 0 & 0 & & 0 & & \\
\hline \multicolumn{7}{|c|}{ Perceived susceptibility to the disease (I am afraid of catching influenza during an epidemic) } \\
\hline Yes & 4320 & 1055 & 24.4 & 3265 & 75.6 & $<0.001$ \\
\hline No & 2786 & 555 & 19.9 & 2231 & 80.1 & \\
\hline Missing & 0 & 0 & & 0 & & \\
\hline \multicolumn{7}{|c|}{ Perceived effectiveness of vaccination (the vaccine can prevent influenza infection) } \\
\hline Yes & 5793 & 1432 & 24.7 & 4361 & 75.3 & $<0.001$ \\
\hline No & 1313 & 178 & 13.6 & 1135 & 86.4 & \\
\hline Missing & 0 & 0 & & 0 & & \\
\hline \multicolumn{7}{|c|}{ Perceived side effects of vaccination (I am scared of the vaccine's side effects) } \\
\hline Yes & 4273 & 921 & 21.6 & 3352 & 78.4 & 0.006 \\
\hline No & 2833 & 689 & 24.3 & 2144 & 75.7 & \\
\hline Missing & 0 & 0 & & 0 & & \\
\hline \multicolumn{7}{|c|}{ Awareness of the free influenza vaccine policy in Beijing } \\
\hline Yes & 5958 & 1483 & 24.9 & 4475 & 75.1 & $<0.001$ \\
\hline No & 1148 & 127 & 11.1 & 1021 & 88.9 & \\
\hline Missing & 0 & 0 & & 0 & & \\
\hline
\end{tabular}

Missing' indicates the number of people who did not answer this question.

${ }^{*} \mathrm{n} / \mathrm{N} \times 100 \%$.

†Pearson's $\chi^{2}$ test.

fUS $\$ 100=680$ yuan.

countries $^{16}$ (25.0\% in the UK, $27.4 \%$ in Germany, $21.8 \%$ in Spain, $24.2 \%$ in France and $24.4 \%$ in Italy), but much lower than in the $\mathrm{USA}^{17}(39.7 \%$ in $2014 / 2015)$. In Beijing, the coverage rate during the 2014/2015 season was consistent with that of $2009 / 2010$ (21.8\%), but higher than that of 2008/2009 and 2010/2011 (16.9\% and $16.7 \%$, respectively), ${ }^{11}$ and an increase in coverage was observed after 2010/2011. In Beijing, influenza immunisation campaigns are conducted each year. Immunisation activities including health education and promotion and better access to vaccination may increase coverage of the influenza vaccine in Beijing. ${ }^{18}$

The present study showed older adults were more likely to be vaccinated than younger adults. The following reasons may contribute to this. First, the free vaccination policy was a key factor. The Beijing government has provided free annual seasonal influenza vaccination to older adults since 2007, and the vaccine coverage rate for this subpopulation increased substantially from $1.7 \%$ during $1999-2004^{19}$ to $48.7 \%$ in the $2014 / 2015$ season. In most regions of China, older adults must pay out of pocket for the seasonal influenza vaccine, which contributed to a very low coverage rate of $1.5 \%-2.2 \%$ between 2004 and 2014. ${ }^{8}$ However, coverage of the influenza vaccine in older adults in Beijing was much lower than that in five Western European countries $(61.1 \%)^{16}$ and the USA $(61.3 \%),{ }^{17}$ and failed to meet WHO's target of $75 \%$ coverage in $2010 .{ }^{16}$ Second, we found older adults were more likely to have a chronic illness than younger ones, while having a chronic illness was positively associated 


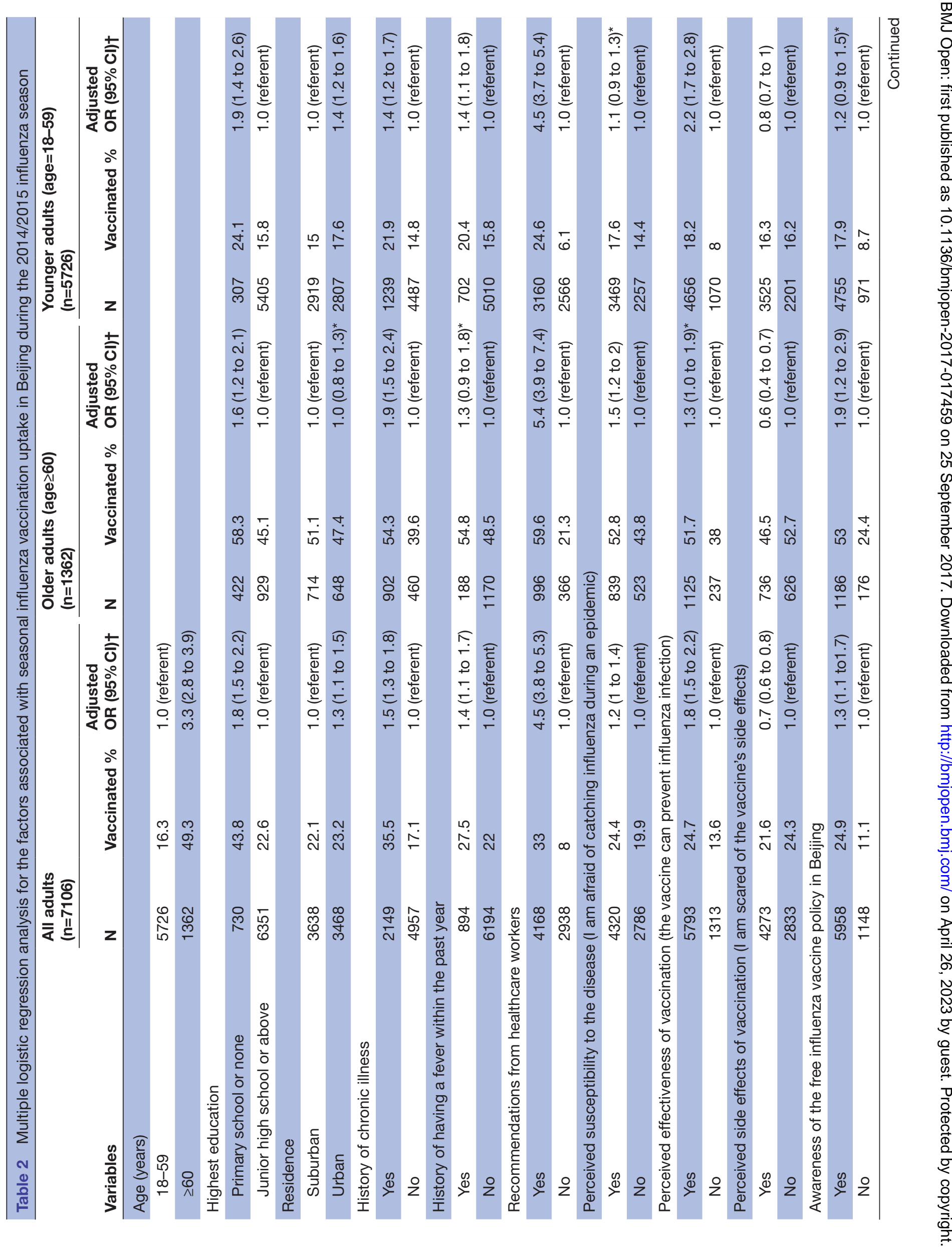


with influenza vaccination uptake in both age groups. This may partly explain the higher vaccination coverage in older adults. Third, age disparity in the coverage rate may also be explained by differing professional recommendations and public perceptions. Although perceived effectiveness of vaccination which was only a predictor for younger adults may have a positive effect on vaccine uptake in younger adults, perceived susceptibility to influenza and awareness of the free influenza vaccine policy were only associated with vaccine uptake in older adults. Moreover, older adults reported receiving recommendations from healthcare professionals more frequently than younger adults, and less likely to be concerned about side effects of the vaccine. These factors affecting vaccine uptake in both age groups may have led to increased uptake.

Recommendations from healthcare workers were the most important factor affecting influenza vaccination uptake in both older and younger adults; previous studies also showed this result. ${ }^{20}{ }^{21}$ Although healthcare workers are the foremost roles who can encourage people to be vaccinated, the vaccination coverage among healthcare workers themselves in Beijing was low. A previous study found only a quarter of healthcare workers received the vaccine against pandemic influenza in the 2009/2010 season, $60 \%$ were concerned about side effects and half had doubts about the vaccine's effectiveness. ${ }^{22}$ Therefore, health promotion activities should be conducted not only for the general population but also for healthcare professionals. More measures should also be taken to motivate these workers to recommend influenza vaccination.

Consistent with two recent meta-analyses, ${ }^{13}{ }^{23}$ the present study documented that public perceptions including concerns about susceptibility to influenza, doubts about the vaccine's effectiveness and fears of side effects can influence the vaccine uptake. Age-related differences were found in perceived susceptibility to influenza and awareness of the free influenza vaccine policy being risk factors for older adults, perceived effectiveness of vaccination for younger adults and perceived side effects of vaccination for both. In the present study, two-fifths did not report fear of catching influenza, $18.5 \%$ did not report the influenza vaccine could prevent infection and $60 \%$ reported fear of side effects. The results indicated that accurate information about the severity of the disease, susceptibility to influenza and vaccine effectiveness and side effects should be conveyed to the public when holding vaccination campaigns in Beijing.

Higher educational attainment is usually considered positively associated with vaccination uptake. ${ }^{24}$ Conversely, we found that lower levels of education had a positive impact on vaccination uptake; a result consistent with our previous study. ${ }^{11}$ In this study, multiple logistic regression analysis by age showed lower education was a risk factor for vaccination in both older and younger adults, and income was not. In other words, influenza vaccination was significantly influenced by educational attainment, independent of age and income. Several reasons may 
contribute to the higher coverage rate among lower-educated people. First, in recent years, media broadcasts and internet discussions targeting vaccine-related adverse outcomes have brought public suspicion in China about influenza vaccination. ${ }^{25}$ Lower-educated people are less likely to be exposed to such information, ${ }^{11}$ and this may have a positive effect on vaccination. Our results, which supported this assumption, showed for both younger and older adults, lower-educated people were less likely to report fear of side effects, but more likely to report viewing influenza as a serious disease. Second, this study found lower-educated older adults more frequently reported receiving recommendations from healthcare workers than did those with higher levels of education. Third, for younger adults, lower-educated people were more likely to have a chronic illness, which was a risk factor for vaccination in this study.

This study found suffering from a fever or chronic illness was associated with greater intent to be vaccinated. That finding was consistent with Blank's study and could be explained by heightened perceptions of personal risk. ${ }^{26}$ We also found younger adults living in urban areas were more likely to receive the vaccine than those from suburban areas, and better access to vaccines may be the main reason for their intent to be vaccinated.

Strengths of this study are its large sample size of 7206 participants and its high response rate of $98.7 \%$, implying the findings are highly representative and reliable. However, there are some limitations in this study. First, considering diversity of income levels and healthcare access across regions, our observations may not be effectively generalisable for other countries or regions. Additionally, because all the information was collected via a self-reported questionnaire, the investigators could not check the accuracy of responses, which may have led to reporting bias. Also, the respondents had to recall their experiences with vaccination; therefore, recall bias cannot be ruled out. Finally, the causal relationships could not be established because of the cross-sectional design.

\section{CONCLUSIONS}

This study demonstrated that the overall coverage rate of the influenza vaccine was relatively low (20.6\%) among adults in Beijing during the 2014/2015 influenza season. For both older and younger adults, recommendations from healthcare workers were positively associated with influenza vaccination uptake and perceived side effects of vaccination had a negative impact. Age-related differences were found in perceived susceptibility to influenza and awareness of the free influenza vaccine policy being the factors affecting vaccine uptake for older adults, and perceived effectiveness of vaccination for younger adults. Apart from free vaccinations for older adults, age disparity in the rate between older and younger adults $(48.7 \%$ vs $16.0 \%)$ may be explained by differing professional recommendations and public perceptions. Vaccination campaigns that target increasing professional recommendations and public perceptions need to be implemented in the coming years.

\section{Author affiliations}

${ }^{1}$ Institute for Infectious Disease and Endemic Disease Control, Beijing Center for Disease Control and Prevention, Beijing, China

${ }^{2}$ Beijing Research Center for Preventive Medicine, Beijing, China

${ }^{3}$ School of Public Health, Capital Medical University, Beijing, China

${ }^{4}$ Dongcheng District Center for Disease Prevention and Control, Beijing, China

${ }^{5}$ Tongzhou District Center for Disease Prevention and Control, Beijing, China

${ }^{6}$ Xicheng District Center for Disease Prevention and Control, Beijing, China

${ }^{7}$ Haidian District Center for Disease Prevention and Control, Beijing, China

${ }^{8}$ Changping District Center for Disease Prevention and Control, Beijing, China

Acknowledgements The authors thank the colleagues from CDCs of Dongcheng, Tongzhou, Xicheng, Haidian, Huairou and Changping District for interviewing the participants, and Adam Goulston, MS, ELS, from Liwen Bianji, Edanz Group China, for editing the English text of a draft of this manuscript.

Contributors SW, JS, PY and QW conceived of the study and participated in its design. SW, JS, HZ, HL, YC, WH, CL and YT collected the data. SW and JS analysed the data. All authors helped to draft the manuscript and have read and approved the final manuscript.

Funding This work was supported by the research funds for Beijing Municipal Science and Technology Commission (D141100003114002), the capital health research and development of special (2014-1-1011), Beijing Health System High Level Health Technology Talent Cultivation Plan (2013-3-098) and Beijing Young Top-notch Talent Project (2014000021223ZK36).

Disclaimer The funders had no role in study design, data collection and analysis, decision to publish or preparation of the manuscript.

Competing interests None declared.

Patient consent Obtained.

Ethics approval This study was approved by the Institutional Review Board and Human Research Ethics Committee of Beijing Center for Disease Prevention and Control.

Provenance and peer review Not commissioned; externally peer reviewed.

Data sharing statement Other data can be requested by emailing the corresponding author.

Open Access This is an Open Access article distributed in accordance with the Creative Commons Attribution Non Commercial (CC BY-NC 4.0) license, which permits others to distribute, remix, adapt, build upon this work non-commercially, and license their derivative works on different terms, provided the original work is properly cited and the use is non-commercial. See: http://creativecommons.org/ licenses/by-nc/4.0/

(c) Article author(s) (or their employer(s) unless otherwise stated in the text of the article) 2017. All rights reserved. No commercial use is permitted unless otherwise expressly granted.

\section{REFERENCES}

1. Feng L, Shay DK, Jiang Y, et al. Influenza-associated mortality in temperate and subtropical Chinese cities, 2003-2008. Bull World Health Organ 2012;90:279-88.

2. World Health Organization. Influenza (Seasonal). http://www.who.int/ mediacentre/factsheets/fs211/en/ (accessed Mar 2017).

3. Jefferson T, Di Pietrantonj C, Rivetti A, et al. Vaccines for preventing influenza in healthy adults. Cochrane Database Syst Rev 2014;3:CD001269.

4. Jefferson T, Di Pietrantonj C, Al-Ansary LA, et al. Vaccines for preventing influenza in the elderly. Cochrane Database Syst Rev 2010;2:CD004876.

5. Jefferson T, Rivetti A, Di Pietrantonj C, et al. Vaccines for preventing influenza in healthy children. Cochrane Database Syst Rev 2012;8:CD004879.

6. Feng L, Yang P, Zhang T, et al. Technical guidelines for the application of seasonal influenza vaccine in China (2014-2015). Hum Vaccin Immunother 2015;11:2077-101.

7. Palache A, Oriol-Mathieu V, Fino $M$, et al. Seasonal influenza vaccine dose distribution in 195 countries (2004-2013): Little progress in estimated global vaccination coverage. Vaccine 2015;33:5598-605. 
8. Yang J, Atkins KE, Feng L, et al. Seasonal influenza vaccination in China: landscape of diverse regional reimbursement policy, and budget impact analysis. Vaccine 2016;34:5724-35.

9. Wang $\mathrm{X}$, Yang $\mathrm{P}$, Seale $\mathrm{H}$, et al. Estimates of the true number of cases of pandemic (H1N1) 2009, Beijing, China. Emerg Infect Dis 2010;16:1786-8.

10. Zhang $Y$, Muscatello DJ, Wang Q, et al. Overview of influenza vaccination policy in Beijing, China: current status and future prospects. J Public Health Policy 2017;38:366-79.

11. Wu S, Yang P, Li H, et al. Influenza vaccination coverage rates among adults before and after the 2009 influenza pandemic and the reasons for non-vaccination in Beijing, China: a cross-sectional study. BMC Public Health 2013;13:636.

12. Zheng $Y$, Yang P, Wu S, et al. A cross-sectional study of factors associated with uptake of vaccination against influenza among older residents in the postpandemic season in Beijing, China. BMJ Open 2013;3:e003662.

13. Yeung MP, Lam FL, Coker R. Factors associated with the uptake of seasonal influenza vaccination in adults: a systematic review. J Public Health 2016;38:746-53.

14. Nguyen T, Henningsen $\mathrm{KH}$, Brehaut JC, et al. Acceptance of a pandemic influenza vaccine: a systematic review of surveys of the general public. Infect Drug Resist 2011;4:197-207.

15. Beijing Bureau of Statistics. Release on major figures of the 2010 national population census in Beijing, China. http://www.bjstats.gov. cn/tjnj/rkpc-2010/indexch.htm (accessed 18 Mar 2017).

16. Blank PR, Schwenkglenks M, Szucs TD. Influenza vaccination coverage rates in five European countries during season 2006/07 and trends over six consecutive seasons. BMC Public Health 2008;8:272.
17. US Centers for Disease Control and Prevention. National early season flu vaccination coverage, United States 2015 http://www.cdc. gov/flu/fluvaxview/1516season.htm (accessed Mar 2017).

18. Heymann DL, Aylward RB. Mass vaccination: when and why. Curr Top Microbiol Immunol 2006;304:1-16.

19. Wu J, Dong ZY, Deng LX, et al. Influenza vaccination practice in Beijing during 1999-2004. J of Pub Health and Prey Med 2005;16:19-21. (in Chinese).

20. Kroneman M, van Essen GA, John Paget W. Influenza vaccination coverage and reasons to refrain among high-risk persons in four European countries. Vaccine 2006;24:622-8.

21. Stinchfield PK. Practice-proven interventions to increase vaccination rates and broaden the immunization season. Am J Med 2008;121:S11-21.

22. Seale $\mathrm{H}$, Kaur $\mathrm{R}$, Wang $\mathrm{Q}$, et al. Acceptance of a vaccine against pandemic influenza $A(\mathrm{H} 1 \mathrm{~N} 1)$ virus amongst healthcare workers in Beijing, China. Vaccine 2011;29:1605-10.

23. Bish A, Yardley L, Nicoll A, et al. Factors associated with uptake of vaccination against pandemic influenza: a systematic review. Vaccine 2011;29:6472-84.

24. Andrew MK, McNeil S, Merry H, et al. Rates of influenza vaccination in older adults and factors associated with vaccine use: a secondary analysis of the Canadian study of health and aging. BMC Public Health 2004;4:36.

25. World Health Organization. Vaccines incident highlights the need for stronger regulation of private vaccines in China. http://www.wpro. who.int/china/mediacentre/releases/2016/20160328/en/ (accessed Mar 2017).

26. Blank PR, Schwenkglenks M, Szucs TD. Disparities in influenza vaccination coverage rates by target group in five European countries: trends over seven consecutive seasons. Infection 2009;37:390-400. 